\title{
Energy Management Strategy Optimization for Application of an Electrical Variable Transmission System in a Hybrid Electric City Bus
}

\author{
Majid Vafaeipour \\ Mohamed El Baghdadi \\ Dai-Duong Tran \\ Joeri Van Mierlo \\ Omar Hegazy* \\ ETEC Department \& MOBI Research Group, \\ Vrije Universiteit Brussel (VUB), Pleinlaan 2, \\ 1050 Brussel, Belgium \\ Flanders Make, 3001 Heverlee, Belgium \\ *Email: omar.hegazy@vub.be
}

\author{
Florian Verbelen \\ Peter Sergeant \\ Department of Electrical Energy, Metals, \\ Mechanical Constructions and Systems, \\ EEDT group, Ghent University, Ghent, \\ Belgium \\ Flanders Make, 3001 Heverlee, Belgium
}

\begin{abstract}
The present study assesses utilization of an Electrical Variable Transmission system for application in the drivetrain of a hybrid electric city bus. To this end, first the corresponding modeling for a hybrid bus topology is performed in presence of a gearbox. Secondly, two optimization-based energy management strategies (EMS) coupled into a Rule-based EMS are examined. The corresponding investigations are performed towards achieving optimized EMSs for efficient power distribution between the drivetrain components. The main outcome of this research addresses improving the fuel consumption while sustaining the State of Charge of the battery for the studied city bus.
\end{abstract}

Keywords-Hybrid electric vehicle; Hybrid electric city bus; Energy management strategy; Electrical variable transmission; Rule-based strategy; Equivalent consumption minimization strategy; Low pass filter; Fuel consumption minimization.

\section{INTRODUCTION}

Hybrid electric city buses are of the most popular means of public transportation considering their higher efficiency and less emission pollution compared to their conventional counterparts. Seeking for proper Energy Management Strategies (EMSs) to properly share the energy between existing power sources (e.g. Internal Combustion Engine (ICE) and battery of electrified vehicles) is an important subject matter attracting the attention of transportation and electrification researchers toward improving the vehicles' performance [1-4]. There have been various studies focusing on the EMS of hybrid city busses throughout the literature. In this regard, Xiong et al. [5] examined an optimal EMS for a post- transmission hybrid electric bus achieving 30\% of energy consumption improvement compared to a conventional bus. In another study and in presence of a blended EMS, convex modeling and optimization were used by HU et al. to examine a tradeoff between the battery downsizing and energy efficiency for a city bus [6]. Hoeijmakers et al. [7], studied a city bus equipped with an Electrical Variable Transmission (EVT) and concluded that the vehicle equipped with EVT consumes less fuel owing to its engine operated in high-efficiency operating points. The previously performed studies also indicate a significant role of EMS control in various HEVs [8-10] not only for improving fuel consumption, but also for preserving battery charge balance. Among various EMSs, the Equivalent Consumption Minimization Strategy (ECMS) has proven as an effective approach [11, 12]. Being derived based on Hamiltonian function of optimal control theory [13] and inspired from Pontryagin's minimum principle [14], ECMS considers the equivalent fuel consumption as a quantitative measure to decide on power sharing of energy sources in HEVs. Along this, several varieties of ECMS have been presented in the literature of this domain. In the earlier studies, e.g. $[15,16]$, driving cycle predictions, performing horizon optimizations besides pattern recognitions incorporated into ECMS have been used. However, the most promising approaches have been relying on instant use of State of Charge (SoC) values as state feedback for dynamic decision making and contrasting its variations towards charge sustaining [1719]. Considering the previous studies, a very promising control approach would be using methods which can combine the merits of Rule-Based (RB) and Optimization-Based $(\mathrm{OB})$ power sharing strategies. 
Hence, such an EMS for an EVT-based city bus application is developed in the present study. In this regard, the power sharing, battery SoC constraints and minimized fuel consumption are considered as main indictors and compared using the ECMS and Low Pass Filter (LPF) power-split techniques both combined with a $\mathrm{RB}$ approach. To this end, first the corresponding modeling for the studied city bus topology equipped with an EVT is performed as a prerequisite for EMS optimizations. Afterwards, the corresponding optimizations are attained in MATLAB/Simulink ${ }^{\mathbb{B}}$ environment via having the established model incorporated into a Genetic Algorithm (GA) optimization scheme, and eventually the results for minimization of fuel consumption are compared.

\section{DRIVETRAIN ARCHITECTURE}

The architecture of an EVT-equipped hybrid electric city bus consists of an inner rotor connected to the ICE and an outer rotor connected to the wheels through their corresponding shafts. The EVT system - thanks to its unique architecture - can bring many advantages to such topology. The possibility of having the wheel and the engine speed decoupled [20] can enhance the vehicle performance as the engine can operate in desired operating points [21]. The net power generated by the engine can partially supply the power required by the wheels while converting the remaining power to electrical form to supply the stator or to be stored directly in the battery [22]. In an EVT-based HEV topology, two electrical ports at the inner rotor and stator are connected to two back-to-back inverters. Fig. 1 illustrates a schematic diagram of an EVT-based HEV topology for a city bus.

\section{MODELING}

To optimize the EMS, first it is needed to establish the vehicle's model before incorporating it into an optimization algorithm. This section goes through the modeling process of the individual components as the subsystems of the vehicle model. The MATLAB/Simulink ${ }^{\circledR}$ environment is used for performing the modeling, simulation and optimization procedures in the present study. Regarding the modeling approach, the backward calculation method [22] is employed since it combines advantages such as simplicity and low computational cost [20] when it comes to integrating the model into an optimization procedure [21].

The required speed and acceleration time series besides vehicle's dynamic parameter inputs (Table I) are used in the vehicle longitudinal dynamics subsystem. To calculate the tractive forces, the subsystem considers

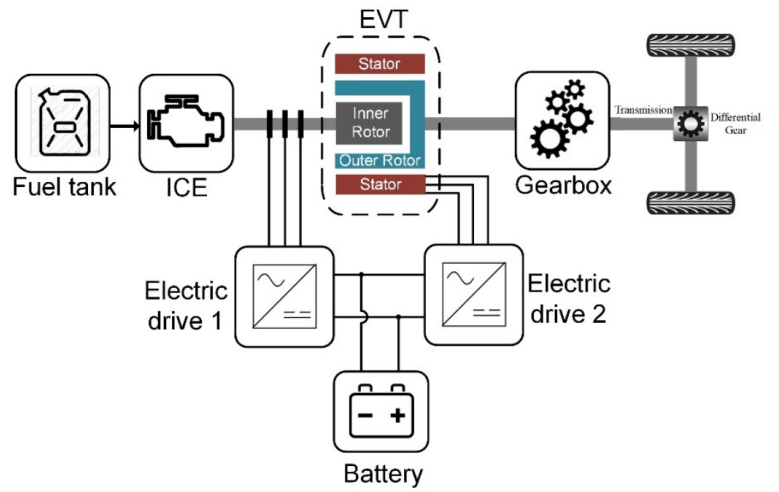

Fig. 1. Schematic of an EVT-equipped hybrid city bus topology.

resistance forces corresponding to drag, wheel rolling, road gradient, and vehicle inertia, road slope and gravitational acceleration as follows:

$F_{T}=\frac{1}{2} \rho v^{2} C_{D} A+C_{r} m g \cos a+m g \sin a+\mathrm{mC}_{\mathrm{J}} \frac{d v}{d t}$

The outputs of the vehicle dynamics subsystem are the wheels' required torque and rotational speed which can be readily calculated knowing the wheels radius and Gear Ratio (GR):

$T_{w}=F_{T} R_{w}(\mathrm{GR})$

$\omega_{w}=\frac{v}{R_{w}}(1 / \mathrm{GR})$

An input-output approach using torque-speed pairs based on the efficiency and the Brake-Specific Fuel Consumption (BSFC) maps are stored into corresponding look-up tables for atypical 140-kW ICE subsystem. In the fuel tank subsystem, the consumed fuel (liter) over the driving cycle can be modelled based on Eq. (4) where $\dot{m}(\mathrm{~g} / \mathrm{s})$ stands for the fuel consumption rate, and $\rho_{f}\left(\mathrm{~kg} / \mathrm{m}^{3}\right)$ represents the fuel's density.

$$
\text { Fuel }=\int_{0}^{t} \frac{m_{f}}{\rho_{f}} d t
$$

For modeling the battery pack, the elements of a firstorder Thevenin equivalent circuit (Fig. 2) as a function of the $\mathrm{SoC}$ were identified by using experimental data [23] and were stored in Simulink ${ }^{\circledR}$ look-up tables. The terminal voltage of the pack $\left(\mathrm{V}_{\text {batt }}\right)$ and $\mathrm{SoC}$ can be mathematically expressed through Eqs. 5-8, where $\mathrm{V}_{\text {oc }}$ is the open circuit voltage, $R_{\text {int }}$ stands for the internal resistance, and $C_{p}$ and $R_{p}$ represent the polarization capacitance and polarization resistance, respectively. A LiFePO4 (LFP) battery type with the specifications given in Table II is considered in this study. 
TABLE I. CONSTANT PARAMETERS FOR VEHICLE DYNAMICS CALCULATIONS.

\begin{tabular}{ccc}
\hline Description & Parameter (unit) & Quantity \\
\hline Mass & $\mathrm{m}(\mathrm{kg})$ & 19000 \\
Drag coefficient & $\mathrm{C}_{\mathrm{D}}$ & 0.56 \\
Rolling resistance & $\mathrm{C}_{\mathrm{r}}$ & 0.005 \\
coefficient & & \\
Rotational inertia & $\mathrm{C}_{\mathrm{J}}$ & 1.08 \\
coefficient & $\mathrm{A}\left(\mathrm{m}^{2}\right)$ & 7.8 \\
Frontal area & $\mathrm{R}_{\mathrm{w}}(\mathrm{m})$ & 0.50 \\
Wheel radius & $\rho\left(\mathrm{kg} / \mathrm{m}^{3}\right)$ & 1.2 \\
Air Density & $\mathrm{GR}$ & 16 \\
Gear Ratio & $g\left(\mathrm{~m} / \mathrm{s}^{2}\right)$ & 9.8 \\
Gravitational acceleration & $a(\mathrm{degree})$ & 0 \\
Road slope & &
\end{tabular}

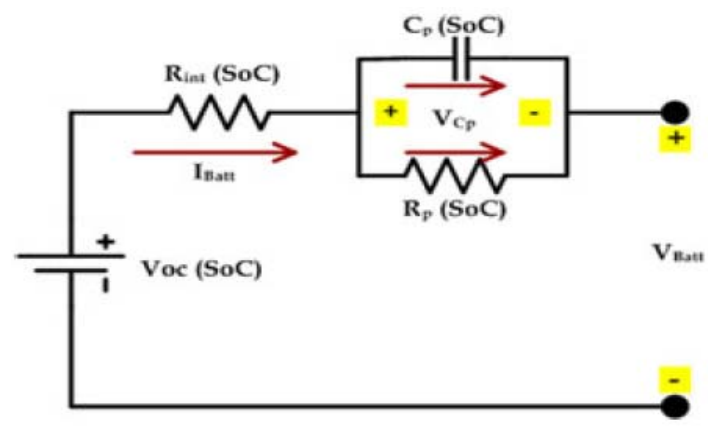

Fig. 2. Thevenin equivalent circuit model [23].

$$
\begin{aligned}
I_{\text {batt }} & =\frac{I_{\text {load }}}{N_{\text {Batt }}} \\
\frac{d V_{c p}}{d t} & =\frac{-V_{c p}}{C_{p} R_{p}}+\frac{I_{\text {Batt }}}{C_{p}} \\
V_{\text {Batt }} & =N_{\text {Bats }}\left(V_{o c}-I_{\text {Batt }} R_{\text {int }}-V_{c p}\right) \\
S o C & =S o C_{0}+\frac{1}{3600} \int \frac{I_{\text {Batt }} d t}{C_{b}}
\end{aligned}
$$

TABLE II. LIFEPO 4 BATTERY CELL SPECIFICATIONS

\begin{tabular}{lc}
\hline Rated capacity & $14 \mathrm{Ah}$ \\
Nominal voltage & $3.6 \mathrm{~V}$ \\
Max discharging current & $100 \mathrm{~A}$ \\
NBatts & 480 \\
SoC 0 & $80 \%$ \\
Min Voltage & 2.5 \\
Max Voltage & 4.15 \\
C_rate limit while charging & -3 \\
\hline
\end{tabular}

Regarding the power converters, the power-efficiency pairs are stored in their corresponding look-up tables and the power flow direction is considered in the calculations. To this end, for the motoring mode (while $\mathrm{P}>0$ ) the efficiency operator $\mu=-1$, and for the braking mode (while $\mathrm{P}<0$ ) the efficiency operator is applied in Eq. (9).

Pout=Pin $\eta^{\mu}$

The EVT rotating field electrical machine subsystem consists of two concentric rotors. The inner rotor encompasses a distributed three-phase winding and the outer rotor is equipped with permanent magnets. The EVT's subsystem model inputs are the inner and outer rotor shafts' torque and speed operating points. These operating conditions are considered and a set of 5 independent currents which minimize the iron and copper losses are correspondingly used in different axes. In this regard, as illustrated in Fig. 3, the stator current is in $\mathrm{d}$ and q-axis, the outer rotor current is in $\mathrm{d}$ axis and the inner rotor current is in $d$ and q-axis [24]. Finite element (FE) calculations validated on a prototype [25] were used to store the results of the corresponding fluxes $(\Psi)$ in the look-up tables used in the EVT subsystem. Knowing the flux and current, the corresponding torque on each component can be calculated as follows, where $\mathrm{N}_{\mathrm{p}}$ is the number of pole pairs and subscripts 1-3 are related to the stator, the outer rotor and the inner rotor, respectively [24-25]:

$$
\begin{aligned}
& T_{1}=\frac{3}{2} N_{p}\left(\psi_{1 q} I_{1 d}-\psi_{1 d} I_{1 q}\right) \\
& T_{2}=-T_{1}-T_{3} \\
& T_{3}=\frac{3}{2} N_{p}\left(\psi_{3 q} I_{3 d}-\psi_{3 d} I_{3 q}\right)
\end{aligned}
$$

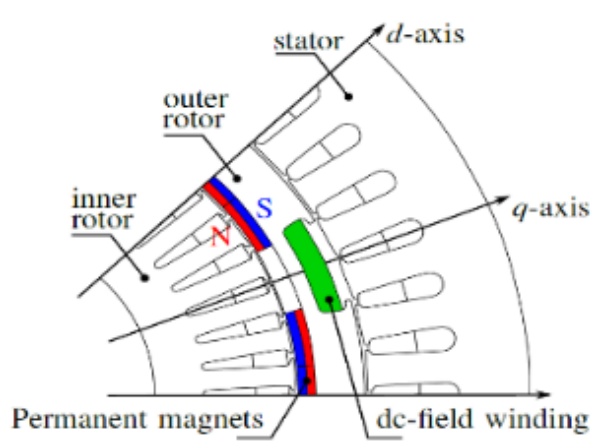

Fig. 3. Cross-sectional view of the PM EVT.

The individually explained subsystems of this section were modeled and integrated in Simulink ${ }^{\circledR}$ environment to form the whole vehicle model as illustrated in Fig. 4. 


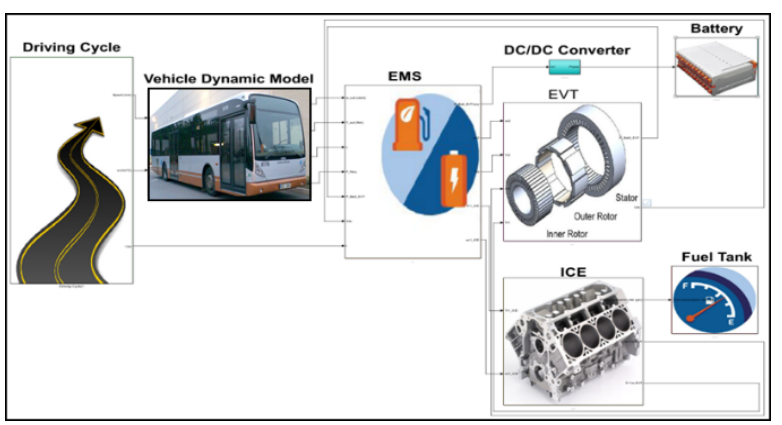

Fig. 4. Diagram of the vehicle backward calculations modeled in Simulink ${ }^{\circledR}$.

\section{ENERGY MANAGEMENT STRATEGY}

The EMS subsystem consisting of a RB scheme combined with two different OB approaches, is the core of power sharing control towards reaching the desired energy management objectives. The main problem of standalone RB strategies is being subjective to predefined perceptions leading to address limited objectives such as sustaining the battery SoC. However, they usually end up to non-optimal solutions regarding objectives such as minimized fuel consumption. Therefore, to provide a robust EMS block, in this study a RB-based strategy is predefined on the top of the EMS and is linked into OB-based Low Pass Filter (LPF) [22, 26] and ECMS control [27] for power sharing as illustrated in Fig. 5.

The optimized EMS selects operating points and modes to satisfy the control objectives. The EMS must deliver the power in a way that the required driving power is fully satisfied. It operates the ICE considering its efficiency map and charges the battery provided not violating the charging limitations. The SoC needs to be sustained between its defined allowable maximum and minimum values providing the initial and final SoC values which are close enough to each other. The objectives and their constraints need to be satisfied while minimizing the fuel consumption. The constraints and objectives considered for the optimization are explained in detail in the next section. To recapitulate the EMS' role, the considered goals are illustrated in Fig. 6.

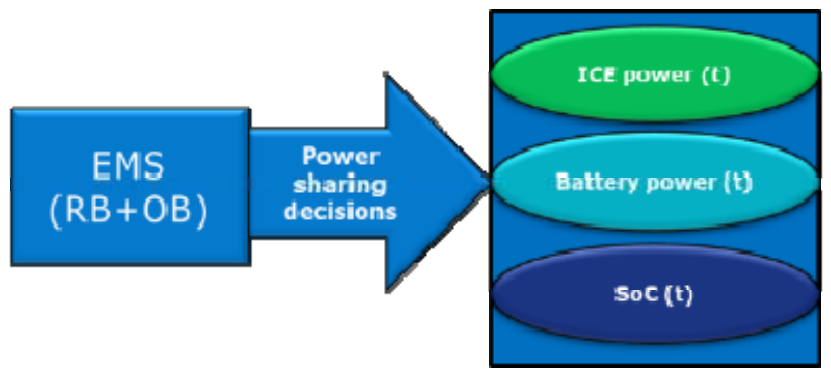

Fig. 5. Power sharing based on RB combined with OB strategy.

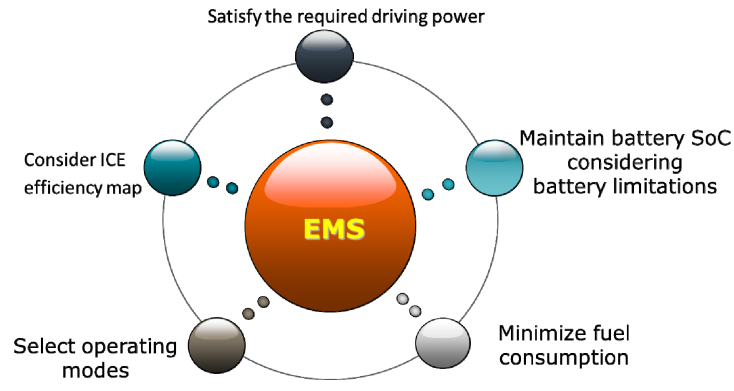

Fig. 6. The EMS objectives.

The rule-based strategy considers a set of "If-ElseThen" rules based on driving patterns, SoC, and requested versus available power. The set of desired objectives can be considered while defining these intuition-based control rules. These rules need to be included as a subsystem of the EMS block to work in tandem with the OB-based power split techniques. The EMS provides a flexible operation of the ICE in efficient operating points to meet the requested driving demands and satisfy the charging requirements. To this end, via using a speed-torque-efficiency lookup table the EMS subsystem rules link the instantaneous split of torque to its corresponding speed leading to the highest efficiency based on the engine map data. The rules update the operating modes considering the status of the demanded loads, required speeds, available energy from sources and $\mathrm{SoC}$ values. The considered modes are categorized as follows and their detailed explanations can be found in the previous works [28-30]:

- Pure-electric mode;

- Hybrid-traction mode;

- Engine-traction and battery-charging mode;

- Hybrid-battery-charging by both ICE and regenerative breaking;

- Regenerative-braking mode.

\section{INCORPORATION OF THE OPTIMIZATION ALGORITHM TO THE MODEL}

GA works based on the evolutionary process concept of natural selection of Darwin's theory. This theory proposes that only the fittest populations can produce offspring through natural selection and survive, while the unsuitable populations will be eliminated. The same concept can be conducted into mathematical optimization where during the processes like crossover, mutation and natural selection, the good design points can be selected while neglecting the worse ones toward finding the objective function solution (survival of the fittest) [30]. The design constraints can be defined separately or be integrated into the objective functions as penalties [31]. In this study the decisive control parameters of the LFP and ECMS strategies [22, 26, 27], 
as the design parameters, are introduced into a MATLAB-based GA incorporated into the Simulinkbased vehicle model. The optimization algorithm and the simulation model work iteratively in tandem for the optimization process. The GA considers the decision variable as the input chromosome besides the defined minimum and maximum values of the constraints to minimize the fuel consumption. The interrelations of the modeling and optimization process are illustrated in Fig. 7. The optimization algorithm searches to minimize the fuel consumption while satisfying the EMS constraints:

$$
\begin{aligned}
& \min (\text { Fuel })=\min J=\min \int_{0}^{t} \frac{m_{f}}{\rho_{f}} d t \\
& \left|S o C_{f}-S o C_{i}\right|<\varepsilon_{0} \\
& S o C_{\text {min }}-\varepsilon<S o C(t)<S o C_{\max }+\varepsilon \\
& C_{-} \text {Rate }(t) \geq \beta
\end{aligned}
$$

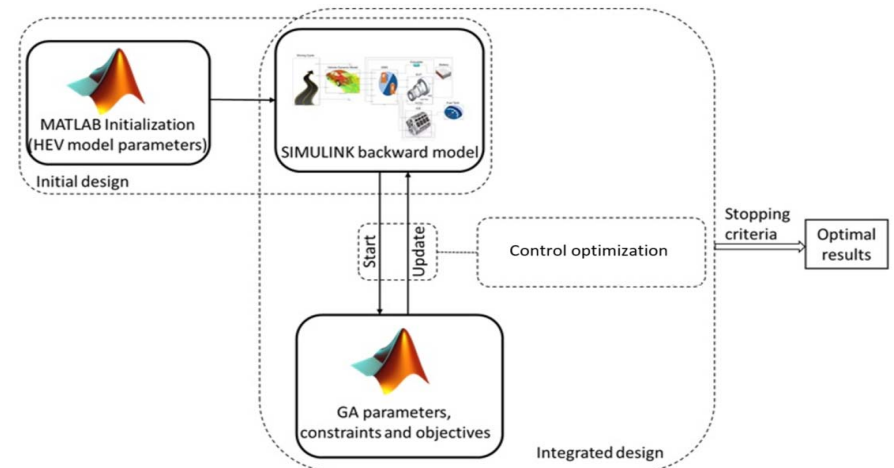

Fig. 7. Coordination of the optimization algorithm and the model.

According to the Eqs. 13-16, two set of SoC constraints are considered where the first one, based on Eq. (14), represents the charge-sustaining requirement in HEVs. The second SoC constraint, based on Eq. (15), expresses the allowable minimum and maximum limits of the SoC considered for the optimization through the driving cycle. The typical charge-sustaining equality constraint $\operatorname{SoC}_{f}=S o C_{i}$ is used to form the inequality constraint (14) to have the, $\Delta S o C$ which is the difference between initial and final $\mathrm{SoC}$ values, can stay within a small feasible boundary $\varepsilon_{0}$ in the optimization process. This constraint can be hardened/softened by altering $\mathcal{E}_{0}$. To avoid sudden charges and to prevent fast aging of the battery pack, the EMS must consider the battery $C_{-}$Rate $(t)$ limitation based on the battery specifications.
In this regard, the $C_{-}$Rate $(t)$ constraints based on Eq. (16) and for the used battery chemistry are considered. For the explained constraints, it is remarkable that they must be incorporated into the optimization process as penalties penalizing the objective function via adding a big enough penalty value when a desired constraint is violated. This method is used to consider the constraints which cannot be directly included in the optimization formulations.

\section{DISCUSSION AND RESULTS}

The results of the simulation and optimizations performed for the studied driving cycle (SORT) are presented and discussed in this section. According to the previous section, the optimized EMSs aim to satisfy the mentioned criteria while minimizing the fuel consumption. Concerning the battery charging criteria, the $C_{-}$Rate $(t)$ must not violate its constraint value denoted as $\beta$. Here, $\beta$ value of -3 based on the $\mathrm{LiFePO}_{4}$ battery chemistry specifications is considered. In addition, for the charge-sustaining constraint, a small enough value of $\varepsilon_{0}=0.3 \%$ for achieving close enough values of the initial and final $\mathrm{SoC}$ are introduced to the algorithm. Regarding the SoC range, values of $75 \%$ for the minimum and $80 \%$ for the maximum are considered, while having $80 \%$ as the initial SoC as well. After performing the optimization, the model is exposed to the optimized EMSs to obtain the power sharing, SoC and $C_{-}$Rate $(t)$ results through the examined driving cycle. As illustrated in Figs. 8 and 9 for the LPF-based and the ECMS-based strategies, the optimized EMSs consider the demanded power to supply the requested load by sharing the power between the engine and the battery while favorably sustaining the battery charge by achieving close enough values of the initial and the final SoC. It also considers the allowable $C_{-}$Rate $(t) \geq-3$ battery limitation. Furthermore, it keeps the $\mathrm{SoC}$ in the defined desired Min and Max window range.

The results indicate that the algorithms have successfully satisfied the control objectives. Table III provides detailed evaluation of the studied cases in terms of the EMS goals, and Table IV compares the obtained average fuel consumptions (liter/100km) over the cycle for each EMS where the ECMS outperforms by $2 \%$. 


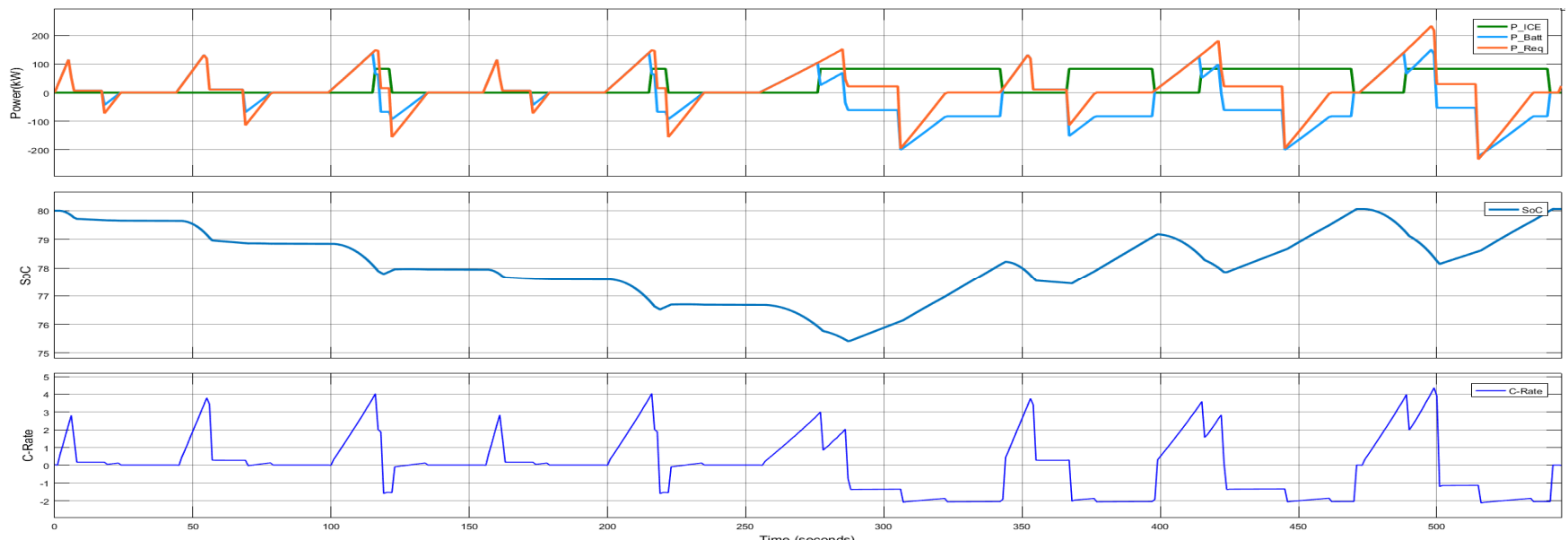

Fig. 8. LPF-based strategy - Power distribution $(\mathrm{kW})$, SoC $(\%)$ recovery, and C-rate results.

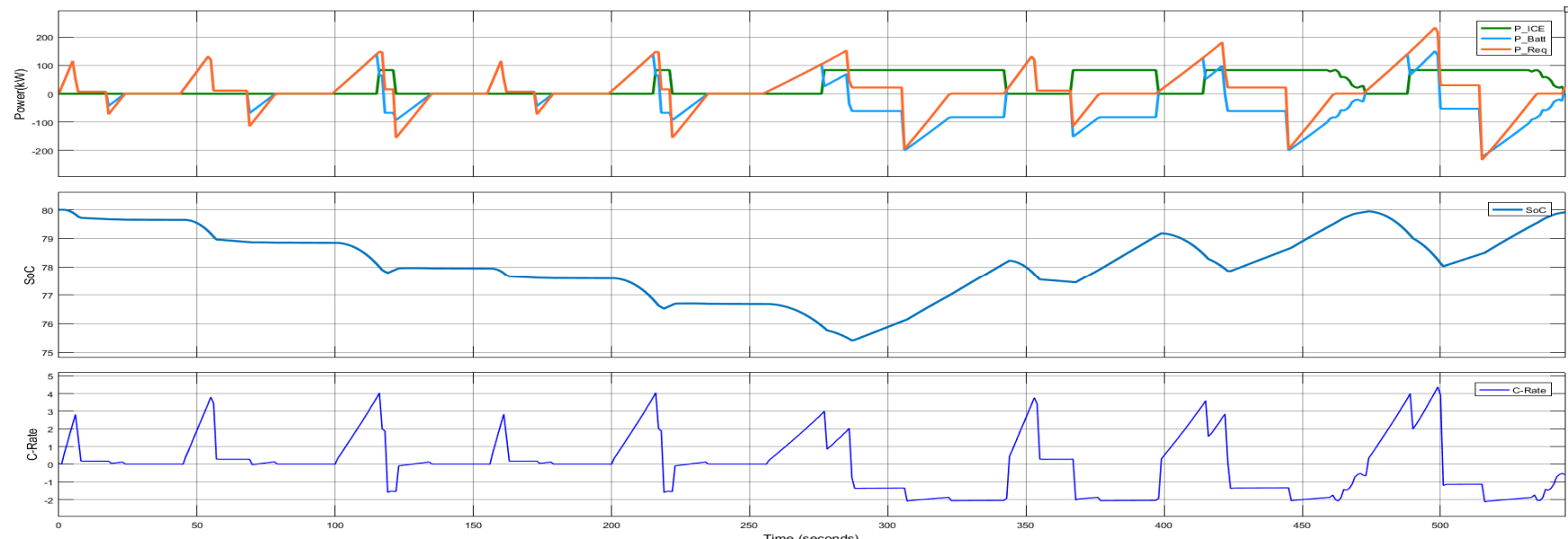

Fig. 9. ECMS-based strategy - Power distribution, SoC (\%) recovery, and C-rate results.

TABLE III. EMS OBJECTIVES EVALUATION

\begin{tabular}{ccc}
\hline Considered features & ECMS & LPF \\
\hline$|\Delta S o C|<0.3 \%$ & $\checkmark$ & $\checkmark$ \\
SoC $\min <$ SoC $<$ SoC $\max$ & $\checkmark$ & $\checkmark$ \\
Driving power needs & $\checkmark$ & $\checkmark$ \\
C_Rate $(t) \geq-3$ & $\checkmark$ & $\checkmark$ \\
All EMS objectives satisfied? & $\checkmark$ & $\checkmark$ \\
\hline
\end{tabular}

TABLE IV. RESULTS OF IMPROVEMENT IN FUEL CONSUMPTION

\begin{tabular}{cccc}
\hline & $\begin{array}{c}\text { Optimized } \\
\text { ECMS+RB }\end{array}$ & $\begin{array}{c}\text { Optimized } \\
\text { LPF+RB }\end{array}$ & Improvement \\
\hline $\begin{array}{c}\text { Fuel Consumption } \\
(\mathbf{L} / \mathbf{1 0 0} \mathbf{~ k m})\end{array}$ & 18.24 & 18.60 & $2 \%$ \\
\hline
\end{tabular}

VII. CONCLUSIONS AND FUTURE WORK DIRECTIONS

The present study investigated two optimized EMSs incorporating the $\mathrm{OB}$ and $\mathrm{RB}$ approaches for a hybrid electric city bus equipped with an EVT system. First the vehicle was modeled in components level and the proposed EMSs were integrated into a GA-based algorithm for the EMS optimization purposes. The power distribution and $\mathrm{SoC}$ results were obtained through the optimization and simulation procedures, while considering battery limitations and EMS objectives. The results have shown that the ECMS-based strategy could improve fuel consumption by $2 \%$ over the LPF-based strategy while both strategies successfully sustained the SoC in a fixed desired range. The integration of the EMS optimization into components optimal sizing is promising for investigations as a future work direction of the present research. One could then investigate possible improvements in fuel consumption besides drivetrain cost reductions for the EVT-based HEV topology. In addition, the successfully simulated EMSs pave the path for their real-time implementation in Hardware-in-the-Loop (HiL) experiments such as the research performed in [32, 33] for validation and consequently utilization in real-world applications.

\section{ACKNOWLEDGMENT}

The authors are grateful to Flanders Make for the support to the current work and to our research group. 


\section{REFERENCES}

[1] D.-D. Tran, M. Vafaeipour, M. El Baghdadi, R. Barrero, J. Van Mierlo, and O. Hegazy, "Thorough state-of-the-art analysis of electric and hybrid vehicle powertrains: Topologies and integrated energy management strategies," Renewable and Sustainable Energy Reviews, p. 109596, 2019.

[2] F. Fazelpour, M. Vafaeipour, O. Rahbari, and M. A. Rosen, "Intelligent optimization to integrate a plug-in hybrid electric vehicle smart parking lot with renewable energy resources and enhance grid characteristics," Energy Conversion and Management, vol. 77, pp. 250-261, 2014.

[3] O. Rahbari, M. Vafaeipour, N. Omar, M. A. Rosen, O. Hegazy, J.-M. Timmermans, et al., "An optimal versatile control approach for plug-in electric vehicles to integrate renewable energy sources and smart grids," Energy, vol. 134, pp. 1053-1067, 2017.

[4] F. Fazelpour, M. Vafaeipour, O. Rahbari, and M. A. Rosen, "Intelligent optimization of charge allocation for plug-in hybrid electric vehicles utilizing renewable energy considering grid characteristics," in 2013 IEEE International Conference on Smart Energy Grid Engineering (SEGE), 2013, pp. 1-8.

[5] W. Xiong, Y. Zhang, and C. Yin, "Optimal energy management for a series-parallel hybrid electric bus," Energy conversion and management, vol. 50, pp. 1730-1738, 2009.

[6] X. Hu, N. Murgovski, L. Johannesson, and B. Egardt, "Energy efficiency analysis of a series plug-in hybrid electric bus with different energy management strategies and battery sizes," Applied Energy, vol. 111, pp. 1001-1009, 2013.

[7] M. J. Hoeijmakers and M. Rondel, "The electrical variable transmission in a city bus," in 2004 IEEE 35th Annual Power Electronics Specialists Conference (IEEE Cat. No. 04CH37551), 2004, pp. 2773-2778.

[8] J. Wu, J. Peng, H. He, and J. Luo, "Comparative analysis on the rule-based control strategy of two typical hybrid electric vehicle powertrain," Energy Procedia, vol. 104, pp. 384-389, 2016.

[9] A. Neffati, S. Caux, and M. Fadel, "Fuzzy switching of fuzzy rules for energy management in HEV," IFAC Proceedings Volumes, vol. 45, pp. 663-668, 2012.

[10] Z. Wei, Z. Xu, and D. Halim, "Study of HEV power management control strategy based on driving pattern recognition," Energy Procedia, vol. 88, pp. 847-853, 2016.

[11] Y.-J. Huang, C.-L. Yin, and J.-W. Zhang, "Design of an energy management strategy for parallel hybrid electric vehicles using a logic threshold and instantaneous optimization method," International Journal of Automotive Technology, vol. 10, pp. 513521, 2009.

[12] B. Wang and Y. Luo, "Application study on a control strategy for a hybrid electric public bus,"
International Journal of Automotive Technology, vol. 12, pp. 141-147, 2011.

[13] S. Delprat, J. Lauber, T.-M. Guerra, and J. Rimaux, "Control of a parallel hybrid powertrain: optimal control," IEEE transactions on Vehicular Technology, vol. 53, pp. 872-881, 2004.

[14] L. Serrao, S. Onori, and G. Rizzoni, "ECMS as a realization of Pontryagin's minimum principle for HEV control," in 2009 American control conference, 2009, pp. 3964-3969.

[15] C. Musardo, G. Rizzoni, Y. Guezennec, and B. Staccia, "A-ECMS: An adaptive algorithm for hybrid electric vehicle energy management," European Journal of Control, vol. 11, pp. 509-524, 2005.

[16] B. Gu and G. Rizzoni, "An adaptive algorithm for hybrid electric vehicle energy management based on driving pattern recognition," in ASME 2006 International Mechanical Engineering Congress and Exposition, 2006, pp. 249-258.

[17] J. T. Kessels, M. W. Koot, P. P. Van Den Bosch, and D. B. Kok, "Online energy management for hybrid electric vehicles," IEEE Transactions on vehicular technology, vol. 57, pp. 3428-3440, 2008.

[18] A. Chasse, A. Sciarretta, and J. Chauvin, "Online optimal control of a parallel hybrid with costate adaptation rule," IFAC proceedings volumes, vol. 43, pp. 99-104, 2010.

[19] S. Onori, L. Serrao, and G. Rizzoni, "Adaptive equivalent consumption minimization strategy for hybrid electric vehicles," in ASME 2010 dynamic systems and control conference, 2010, pp. 499-505.

[20] F. Millo, L. Rolando, and M. Andreata, "Numerical simulation for vehicle powertrain development," in Numerical Analysis-Theory and Application, ed: IntechOpen, 2011.

[21] M. Chehresaz, "Modeling and design optimization of plug-in hybrid electric vehicle powertrains," University of Waterloo, 2013.

[22] M. Vafaeipour, M. El Baghdadi, F. Verbelen, P. Sergeant, J. Van Mierlo, K. Stockman, et al., "Technical assessment of utilizing an electrical variable transmission System in hybrid electric vehicles," in 2018 IEEE Transportation Electrification Conference and Expo, Asia-Pacific (ITEC Asia-Pacific), 2018, pp. 1-5.

[23] O. Hegazy, R. Barrero, J. Van Mierlo, P. Lataire, N. Omar, and T. Coosemans, "An advanced power electronics interface for electric vehicles applications," IEEE transactions on power electronics, vol. 28, pp. 5508-5521, 2013.

[24] J. Druant, H. Vansompel, F. De Belie, and P. Sergeant, "Optimal control for a hybrid excited dual mechanical port electric machine," IEEE Transactions on Energy Conversion, vol. 32, pp. 599607, 2017.

[25] J. Druant, H. Vansompel, F. De Belie, J. Melkebeek, and P. Sergeant, "Torque analysis on a double rotor electrical variable transmission with hybrid 
excitation," IEEE Transactions on Industrial Electronics, vol. 64, pp. 60-68, 2016.

[26] M. Vafaeipour, D.-D. Tran, M. El Baghdadi, F. Verbelen, P. Sergeant, K. Stockman, et al., "Optimized Energy Management Strategy for a HEV Equipped with an Electrical Variable Transmission System," in 32nd Electric Vehicle Symposium (EVS32), 2019.

[27] M. Vafaeipour, M. El Baghdadi, J. Van Mierlo, O. Hegazy, F. Verbelen, and P. Sergeant, "An ECMSbased approach for energy management of a HEV equipped with an electrical variable transmission," in 2019 Fourteenth International Conference on Ecological Vehicles and Renewable Energies (EVER), 2019, pp. 1-9.

[28] J. Van Mierlo and O. Hegazy, "Series Hybrid Electric Vehicles (SHEVs)," Encyclopedia of Automotive Engineering, pp. 1-12, 2014.

[29] J. Van Mierlo and O. Hegazy, "Parallel Hybrid Electric Vehicles (Parallel HEVs)," Encyclopedia of Automotive Engineering, pp. 1-10, 2014.

[30] C. Mi and M. A. Masrur, Hybrid electric vehicles: principles and applications with practical perspectives: John Wiley \& Sons, 2017.

[31] M. Montazeri-Gh and A. Poursamad, "Application of genetic algorithm for simultaneous optimisation of HEV component sizing and control strategy," International Journal of Alternative Propulsion, vol. 1, pp. 63-78, 2006.

[32] M. Vafaeipour, M. El Baghdadi, F. Verbelen, P. Sergeant, J. Van Mierlo, and O. Hegazy, "Experimental Implementation of Power-Split Control Strategies in a Versatile Hardware-in-theLoop Laboratory Test Bench for Hybrid Electric Vehicles Equipped with Electrical Variable Transmission," Applied Sciences, vol. 10, p. 4253, 2020.

[33] F. Verbelen, W. Lhomme, E. Vinot, J. Stuyts, M. Vafaeipour, O. Hegazy, et al., "Comparison of an optimized electrical variable transmission with the Toyota Hybrid System," Applied Energy, vol. 278, p. $115616,2020$. 